\title{
Chlorinated Polyfluoroalkyl Ether Sulfonic Acids in Marine Organisms from Bohai Sea, China: Occurrence, Temporal Variations, and Trophic Transfer Behavior
}

\author{
Yanwei Liu, ${ }^{\dagger, \ddagger}$ Ting Ruan, ${ }^{\dagger, \ddagger}$ Yongfeng Lin, ${ }^{\dagger, \ddagger}$ Aifeng Liu, ${ }^{\dagger, \ddagger}$ Miao Yu, ${ }^{\dagger \neq}$ Runzeng Liu, ${ }^{\dagger, \ddagger}$ Mei Meng,

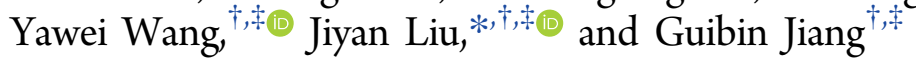 \\ ${ }^{\dagger}$ State Key Laboratory of Environmental Chemistry and Ecotoxicology, Research Center for Eco-Environmental Sciences, Chinese \\ Academy of Sciences, Beijing 100085, China \\ ${ }^{\ddagger}$ College of Resources and Environment, University of Chinese Academy of Sciences, Beijing 100049, China
}

Supporting Information

ABSTRACT: F-53B, the commercial product of chlorinated polyfluoroalkyl ether sulfonic acids (Cl-PFESAs), has been used in Chinese chrome plating industry for 30 years, and was recently identified in the environment, which caused great concerns. So far, limited investigations have been performed on their environmental occurrence, fate and impact. In this study, we demonstrated the wide occurrence of Cl-PFESAs and their trophic transfer behavior in marine organisms from Chinese Bohai Sea. 6:2 Cl-PFESA ( $<0.016-0.575 \mathrm{ng} / \mathrm{g}$ wet weight $)$ was the dominant congener, and 8:2 Cl-PFESA $(<0.022-0.040 \mathrm{ng} /$ g) was occasionally detected. Compared to other perfluoroalkyl and polyfluoroalkyl substances (PFASs) of concern, the levels

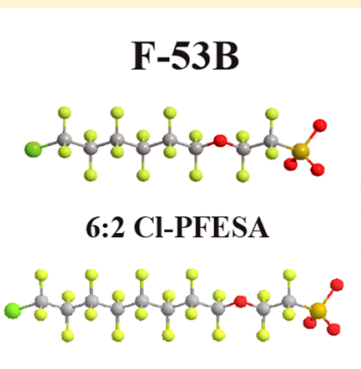

8:2 CI-PFESA

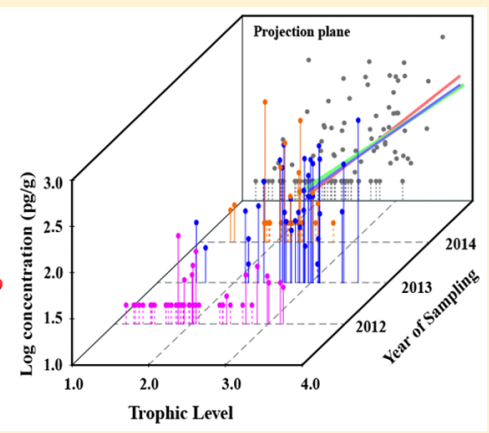
of Cl-PFESAs were relatively lower in marine organisms. Based on the comparative analysis of Cl-PFESA contamination in mollusk samples collected in 2010-2014, both the concentrations and detection frequencies of Cl-PFESAs tended to increase in this region. And this kind of chemicals were more vulnerable to be accumulated in marine organisms at relatively higher trophic levels. Similar to perfluorooctanesulfonate (PFOS) and the long chain perfluorinated carboxylates (PFCAs), 6:2 Cl-PFESA could be magnified along the food chain. Accordingly, the potential threat might be posed to the wildlife and human beings due to unintended exposure to Cl-PFESAs.

\section{INTRODUCTION}

Chlorinated polyfluoroalkyl ether sulfonic acids (Cl-PFESAs), with the trade name of F-53B, have been applied as the mist suppressant to prevent the emissions of the toxic substance, chromium, for decades in Chinese chrome plating industry. ${ }^{1,2}$ The annual production of F-53B was estimated to be $20-30$ tons in China. ${ }^{3}$ As we know, perfluorooctanesulfonate (PFOS) and its derivatives were widely used as mist suppressants before this compound was listed into the Stockholm Convention on Persistent Organic Pollutants (POPs) in 2009. Due to the similar structure and physicochemical properties to PFOS, F$53 \mathrm{~B}$, as the potential PFOS alternative, would probably be increasingly produced and extensively applied., ${ }^{1,2,4}$

Although the production and application of Cl-PFESAs have been started since $1970 \mathrm{~s},{ }^{5}$ specific concerns on their environmental occurrence and behavior have not arisen until recently. Several studies have ascertained the omnipresence of $\mathrm{Cl}$ PFESAs in Chinese environment. ${ }^{1-3,6}$ The two major components of the commercial F-53B, 6:2 Cl-PFESA and 8:2 Cl-PFESA, were found in fish samples collected from Tangxun Lake, and human serum and urine samples from China. ${ }^{6}$ 6:2 ClPFESA was suggested to be the most persistent perfluoroalkyl and polyfluoroalkyl substances (PFASs) in humans, ${ }^{6}$ and its concentrations were comparable to those of PFOS in influent and effluent from the wastewater treatment plant (WWTP) in China. ${ }^{2}$ Empirical data on surface water of 19 Chinese rivers and municipal sewage sludge samples revealed a relatively high Cl-PFESA contamination in Eastern China. ${ }^{1,7}$ 6:2 Cl-PFESA was also determined in marine mammals collected from East Greenland, such as polar bears, ringed seals and killer whales. ${ }^{8}$ Although the pollution source and transportation pathway of 6:2 Cl-PFESA in Arctic remained unclear, this finding suggested the potential worldwide contamination of $\mathrm{Cl}$ PFESAs.

Cl-PFESAs showed similar distribution profiles, enrichment mechanisms, biological toxicity and elimination kinetics to the well-known PFASs, like PFOS. ${ }^{1-3,6-9}$ For example, positive correlations were reported between the concentrations of 6:2 Cl-PFESA and PFOS in sewage sludge, marine mammals and

Received: December 29, 2016

Revised: March 19, 2017

Accepted: March 19, 2017

Published: March 20, 2017 
human beings, indicating their common pollution sources and/ or similar environmental behaviors. ${ }^{1,6,8}$ Comparable air-water, octanol-water, and octanol-air partition coefficients $\left(K_{\mathrm{aw}}, K_{\mathrm{ow}}\right.$, and $K_{\mathrm{oa}}$ ) were obtained for the molecular forms of 6:2 ClPFESA and PFOS by COSMOtherm. ${ }^{4}$ PFOS and several long chain perfluorinated carboxylates (PFCAs) have been verified to be bioaccumulated and biomagnified in marine and freshwater ecosystems. ${ }^{10-15}$ Similarly, 6:2 Cl-PFESA was found to be highly bioaccumulated in crucian carps. ${ }^{3}$ However, the investigation on the fate of Cl-PFESAs in organisms, especially along the food chains, is very limited. The lack of field research on Cl-PFESAs in the marine ecosystem could impede the accurate evaluation of their potential ecological and health risks.

In this study, we investigated the concentrations of $\mathrm{Cl}$ PFESAs in 114 biota samples, containing 8 fishes, 11 invertebrates, and 2 algae species, which were collected from Chinese Bohai Sea with the inflows from over 40 rivers. The compounds of PFCAs, perfluorinated sulfonates (PFSAs) and fluorotelomer sulfonates (FTSAs) were simultaneously investigated to clarify the environmental behavior of Cl-PFESAs. Our objectives are to (1) recognize the contamination level of Cl-PFESAs and their congener profile in marine organisms, (2) characterize their spatial and temporal variations in the Bohai Sea, and (3) investigate their trophic transfer behavior.

\section{EXPERIMENTAL SECTION}

Chemicals and Reagents. The 6:2 Cl-PFESA and 8:2 ClPFESA standards were laboratory-purified as described in our previous work. ${ }^{1}$ Other standard solutions were purchased from Wellington Laboratories, including a native PFASs standard mixture (PFAC-MXB, $2 \mu \mathrm{g} / \mathrm{mL}$ ), fluorotelomer sulfonates standards $(4: 2,6: 2$ and 8:2 FTSA, $50 \mu \mathrm{g} / \mathrm{mL})$ and isotopelabeled surrogate standards (MPFAC-MXA, $2 \mu \mathrm{g} / \mathrm{mL}$ for each analyte; M2-6:2 FTSA, $50 \mu \mathrm{g} / \mathrm{mL}$ ). All stock standards and solutions were prepared in methanol and stored at $4{ }^{\circ} \mathrm{C}$.

HPLC grade methanol was purchased from J.T. Baker (Phillipsburg, NJ). HPLC formic acid and ammonium acetate were obtained from Dikma (Lake Forest, CA). Sodium hydroxide was purchased from Sinopharm Chemical Reagent, Inc. (Beijing, China). Ammonium hydroxide (analytical grade; $v / v, 50 \%)$ was purchased from Alfa Aesar (Ward Hill, MA). Deionized water was prepared using a Milli-Q system (Millipore, Billerica, MA) with a resistance of $18.3 \mathrm{M} \Omega \cdot \mathrm{cm}^{-1}$.

Sample Collection and Preparation. Bohai Sea is a semienclosed inland sea of China, surrounded by Liaodong Peninsula, the North China Plain and Shandong Peninsula. It is endowed with coastal and marine resources, such as fisheries, oil and gas. The coastal region around Bohai Sea is highly industrialized. Therefore, environmental contamination in this region has attracted public attention. Biological samples were collected along the coastline of Bohai Sea in 2010-2014. The sampling information, including organism species, size, site, time and some other details, is shown in Supporting Information (SI) Figure S1 and Table S1.

The sample collection, transport and preparation have been previously reported. ${ }^{16,17}$ In brief, the collected samples were transported to the laboratory on ice. All samples were cleaned by tap water and Milli-Q water to remove extraneous impurities. Stainless steel scalpel blades were used to excise target tissues. The whole bodies of algae, soft tissue of invertebrates, and flesh of fish were submitted to chemical analysis. A certain number of individuals were combined into one composite sample. The details of the composite samples were given in SI Table S1. Finally, samples were wrapped with aluminum foil and stored in zip locked bags at $-20{ }^{\circ} \mathrm{C}$ until analysis. All equipment and storage containers were rinsed with water and acetone before use to avoid cross contamination.

Sample Pretreatment. A modified alkaline digestion method was used for sample pretreatment. ${ }^{18}$ In brief, $0.2 \mathrm{~g}$ tissue samples were spiked with surrogate standards (1 ng MPFAC-MXA and $1 \mathrm{ng} \mathrm{M2-6:2} \mathrm{FTSA),} \mathrm{mixed} \mathrm{with} 10 \mathrm{~mL}$ of methanol containing $10 \mathrm{mM} \mathrm{NaOH}$, and then vigorously shaken at $250 \mathrm{rpm}$ (round per minute) for $16 \mathrm{~h}$. After extraction and centrifugation (3000 rpm, $10 \mathrm{~min})$, the supernatants were diluted to $100 \mathrm{~mL}$ with Milli-Q water, and then loaded onto Oasis WAX SPE cartridges (6 cc, $150 \mathrm{mg}$; Waters Corp. Milford, MA) which were preconditioned with 4 $\mathrm{mL}$ of methanol containing $0.1 \%$ ammonium hydroxide $(v / v)$, $4 \mathrm{~mL}$ of methanol, and $4 \mathrm{~mL}$ of $0.1 \mathrm{M}$ formic acid water solution at a rate of $1-2 \mathrm{drop} / \mathrm{s}$ in sequence. Cartridges were then washed with $4 \mathrm{~mL}$ of methanol to remove impurities, dried under vacuum for $30 \mathrm{~min}$ and centrifuged at $5000 \mathrm{rpm}$ for $10 \mathrm{~min}$ to remove the residual water. After that, $6 \mathrm{~mL}$ of methanol containing $0.1 \%$ ammonium hydroxide was used to elute target compounds. Finally, the eluate was concentrated to $200 \mu \mathrm{L}$, and centrifuged at $10000 \mathrm{rpm}$ to remove particulate impurities before injection.

Instrumental Analysis. An API 5500 triple-quadrupole mass spectrometer ( $\mathrm{AB}$ SCIEX Inc., Framingham, MA) coupled with an Ultimate 3000 ultrahigh performance liquid chromatograph (Thermo Fisher Scientific Inc., Waltham, MA) was employed for chemical analysis. Electrospray ionization (ESI) was performed in the negative ion multiple reaction monitoring (MRM) mode. Capillary voltage and ionization source temperature were $3000 \mathrm{~V}$ and $500{ }^{\circ} \mathrm{C}$. The separation of the compounds was achieved by an ACQUITY UPLC BEH C18 analytical column $(2.1 \mathrm{~mm}$ i.d. $\times 100 \mathrm{~mm}$ length, $1.7 \mu \mathrm{m}$, Waters Corp. Milford, MA). The mobile phase consisted of methanol and water (containing $1 \mathrm{mM} \mathrm{NH}_{4} \mathrm{Ac}$ additive in both solvents) was initially controlled at ratio of 70:30 (held for 1 $\mathrm{min}$ ), then switched to $100 \%$ methanol in $6 \mathrm{~min}$ (held for 5 $\mathrm{min}$ ), and finally decreased to 70:30 in another $5 \mathrm{~min}$. The flow rate, injection volume and column temperature were set at 0.4 $\mathrm{mL} / \mathrm{min}, 5 \mu \mathrm{L}$ and $40{ }^{\circ} \mathrm{C}$, respectively. Information on parent and daughter ions, corresponding isotope-labeled standards and molecular structures of target compounds are summarized in SI Table S2 and Figure S2.

Quality Assurance and Quality Control (QA/QC). Solvent blanks were injected every three samples, and no carryover effect was observed. Two procedural blanks were included in each batch of seven biological samples to check possible laboratory contamination and interference. The analytes were identified by comparing the retention time and the relative abundance of monitored ions with the standards. Nine-point calibration curves with the concentration range of $0-20 \mathrm{ng} / \mathrm{mL}$ were routinely conducted to determine sample concentrations, showing good linear correlation coefficients $(R$ $>0.998)$. The method detection limit (MDL) was defined as the lowest concentration of a chemical in the sample whose response equaled 3 times noise of the baseline, that is, signal-tonoise ratio $(\mathrm{S} / \mathrm{N})$ was 3 . For the analytes detected in procedural blanks, MDLs were determined as the mean blank concentration plus three times standard deviation. Recoveries of the isotope-labeled surrogate standard for Cl-PFESAs were in the range of $52-71 \%$. All concentrations in this study were 
based on wet weight (ww), and corrected by procedural blanks and the recoveries of isotope-labeled surrogate standards.

Stable Isotope Analysis and the Related Calculation. A Thermo DELTA V Advantage isotope ratio mass spectrometer interfaced to a Flash EA1112 HT elemental analyzer (Thermo Fisher) was applied to determine stable isotope of nitrogen $\left(\delta^{15} \mathrm{~N}\right)$ and carbon $\left(\delta^{13} \mathrm{C}\right)$ (SI Table S1). Lipid normalized $\delta^{13} \mathrm{C}$ values in fish and invertebrates were in the range from $-22.59 \%$ to $-14.71 \%$, which were consistent with the previous data $(-25.38 \% 0$ to $-11.08 \% 0)$ determined in a Bohai food web. ${ }^{19,20}$ The $\delta^{13} \mathrm{C}$ values of a species collected from different sites were very close, implying their similar food composition. The values of $\delta^{15} \mathrm{~N}$ were used to calculate the trophic levels of samples. As the filter feeders were often used as the reference for the trophic position, ${ }^{21-23}$ the trophic level (TL) of zhikong scallop (Chlamys farreri) was considered to be 2.0 in this study and the following equation was used to calculate the TLs of the other marine biota. ${ }^{24}$

$$
\mathrm{TL}_{\text {organism }}=\left(\delta^{15} \mathrm{~N}_{\text {organism }}-\delta^{15} \mathrm{~N}_{\text {Chlamysfarreri }}\right) / 3.8+2.0
$$

Where, $\mathrm{TL}_{\text {organism }}$ refers to the trophic level of an organism, $\delta^{15} \mathrm{~N}_{\text {Chlamys farreri }}$ is 8.92, and 3.8 is the trophic enrichment factor of $\delta^{15} \mathrm{~N}$.

The calculation of trophic magnification factors (TMFs) in the food webs was based on the linear correlation between the contaminant concentrations and the trophic levels of biota. TMFs were obtained by the following equations:

$$
\begin{aligned}
& \log C=a \times \mathrm{TL}+k \\
& \mathrm{TMF}=10^{a}
\end{aligned}
$$

Where, $C$ represents the concentration of the target compound, and $k$ is a constant. It is commonly considered that the compound has biomagnification potential if TMF $>1$.

Statistical Analysis. R statistical language (version 3.3.1), Minitab 17 and Microsoft Excel were used for data analysis according to the recommendation of Helsel. ${ }^{26}$ The $t$-test, sign test and generalized Wilcoxon test were applied to evaluate the effects of different variables. Nonparametric Spearman's rho coefficient was used to evaluate the correlation of data. The statistical calculations described above were conducted for the analytes with detection frequencies higher than $50 \%$. To avoid the biased regression results, maximum likelihood estimation (MLE), and substitution nondetectable data with the values of half MDLs and those generated by Regression on Order Statistics (ROS) were performed for TMF calculation, and the results were compared. ${ }^{27}$ The robust ROS and MLE methods were valid, when the censored data were no more than $80 \%$ of the entire data set. ${ }^{27}$

\section{RESULTS AND DISCUSSION}

\section{Cl-PFESA Concentrations in Marine Organisms.} Detection frequencies, MDLs and concentrations of analytes are summarized in SI Table S3 and S4. The detection frequencies of 8:2 Cl-PFESA, FTSAs, PFBS, and PFDS were lower than $18 \%$. 8:2 Cl-PFESA was detectable only in five species, octopus, mantis shrimp, branded goby, ark shell and venus clam (Figure 1). Nevertheless, 6:2 Cl-PFESA, PFHxS, PFOS, PFNA, PFDA, PFUnDA, and PFTrDA showed relatively high detection frequencies. 6:2 Cl-PFESA was detected in nearly all organism species with the detection frequency of $50 \%$, indicating its wide occurrence in Bohai marine ecosystem. The concentrations of 6:2 Cl-PFESA were

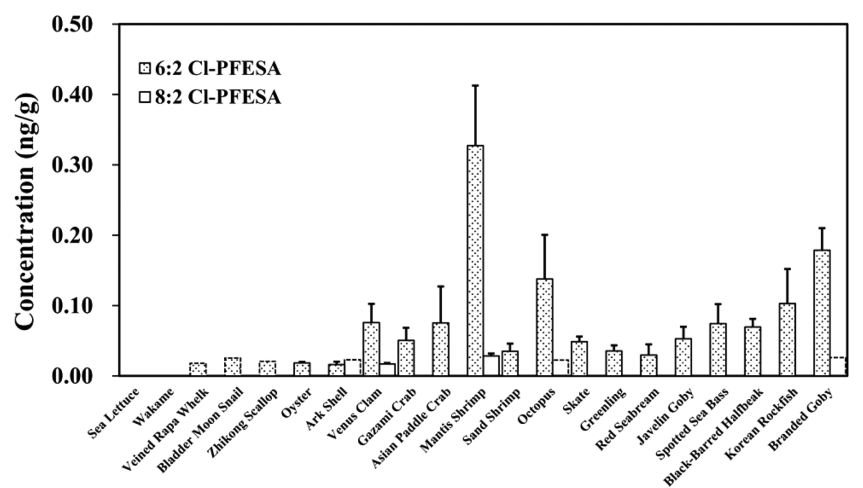

Figure 1. 6:2 Cl-PFESA and 8:2 Cl-PFESA concentrations (mean \pm standard error) in various marine organisms from Bohai Sea, China. Bars in dash line represent the possible highest mean concentration when ROS mean was not able to be calculated due to too many censored data $(>80 \%)$. Concentrations below MDLs were not shown.

significantly higher $(<0.016-0.575 \mathrm{ng} / \mathrm{g})$ than those of $8: 2 \mathrm{Cl}$ PFESA $(<0.022-0.040 \mathrm{ng} / \mathrm{g})$ in all marine organisms (generalized Wilcoxon test, $p<0.001$ ), which was consistent with F-53B component profiles in the commercial products, sludge samples, fish and human serum. ${ }^{1,6}$ The concentration of 6:2 Cl-PFESA in mantis shrimp was the highest (mean \pm standard error: $0.328 \pm 0.085 \mathrm{ng} / \mathrm{g}$ ), followed by those in branded goby muscle $(0.179 \pm 0.031 \mathrm{ng} / \mathrm{g})$ and octopus $(0.138$ $\pm 0.063 \mathrm{ng} / \mathrm{g})$. Apparently, 6:2 Cl-PFESA concentrations in marine animals from Bohai Sea were at the same magnitude when compared with the previously reported observations in polar bears $(0.27 \pm 0.04 \mathrm{ng} / \mathrm{g})$, ringed seals $(0.023 \pm 0.009 \mathrm{ng} /$ $\mathrm{g})$ and killer whales $(0.023 \pm 0.009 \mathrm{ng} / \mathrm{g})$ in East Greenland. ${ }^{8}$ However, 6:2 Cl-PFESA levels in marine fish muscle from Bohai Sea $(<0.016-0.221 \mathrm{ng} / \mathrm{g})$ were lower than those in freshwater fish muscle samples from Tangxun Lake (0.770$2.17 \mathrm{ng} / \mathrm{g}$ ) and Xiaoqing River (1.29-4.83 ng/g).,

Figure 2 illustrates that the concentrations of 6:2 Cl-PFESA and PFOS in shrimp, cephalopod, crab and fish were significantly higher than those in bivalve, gastropod and algae (generalized Wilcoxon test, $p<0.05$ ). The concentration of $6: 2$ Cl-PFESA in fish muscle samples was lower (but not significant) than that in mantis shrimp and cephalopod though these organisms displayed approximate trophic levels. According to the analysis of 6:2 Cl-PFESA distribution in crucian carp from Xiaoqing River and Tangxun Lake, the chemical concentrations in muscle (median: 3.01 and $0.76 \mathrm{ng} / \mathrm{g}$ ) were relatively lower than those in blood (41.9 and $20.9 \mathrm{ng} / \mathrm{g}$ ), kidney (24.64 and $10.34 \mathrm{ng} / \mathrm{g}$ ) and other tissues. ${ }^{3}$ Therefore, it was suspected that the concentrations of 6:2 Cl-PFESA in the whole body of fish from Bohai Sea could be higher than the values in the muscle detected in this study. According to online information from FishBase, ${ }^{28}$ all selected fish species in this study lived in the demersal or reef-associated environment, mainly feeding on invertebrates and small fish, while their migration behaviors were different (SI Table S1). This might explain the results in Figure 3, that was, 6:2 Cl-PFESA concentrations in diverse fish species followed the order of oceanodromous fish (TL: $3.1 \pm 0.2$ ) < diadromous fish (TL: $2.6 \pm 0.3)<$ nonmigratory fish (TL: $3.0 \pm 0.2$ ), which was different from the order of their trophic levels. Similar patterns were observed for PFHxS, PFOS, PFHxA, PFDA, and PFTrDA. Although the trophic levels of nonmigratory fish and oceanodromous fish were quite similar, the concentrations 

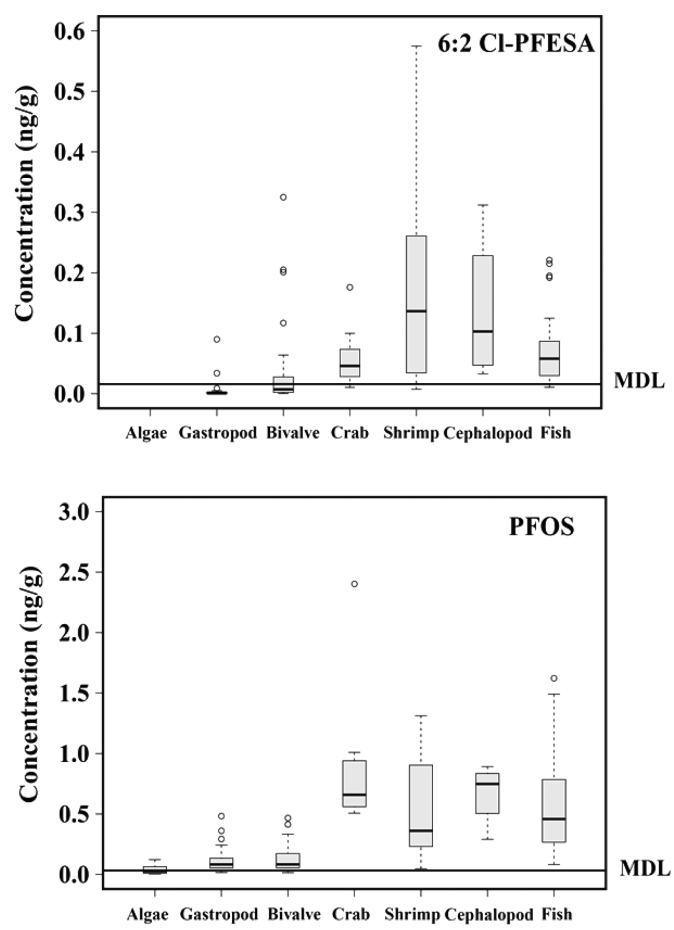

Figure 2. Boxplot of 6:2 Cl-PFESA and PFOS concentrations in different marine organisms from Bohai Sea, China. The horizontal line represents the method detection limit (MDL). 6:2 Cl-PFESA was undetectable in algae samples. The horizontal line in the box represents the median concentration. The edges of the box display the 25th and 75th percentiles. The whiskers display fifth and 95th percentiles. ${ }^{\circ}$ specifies outliers.

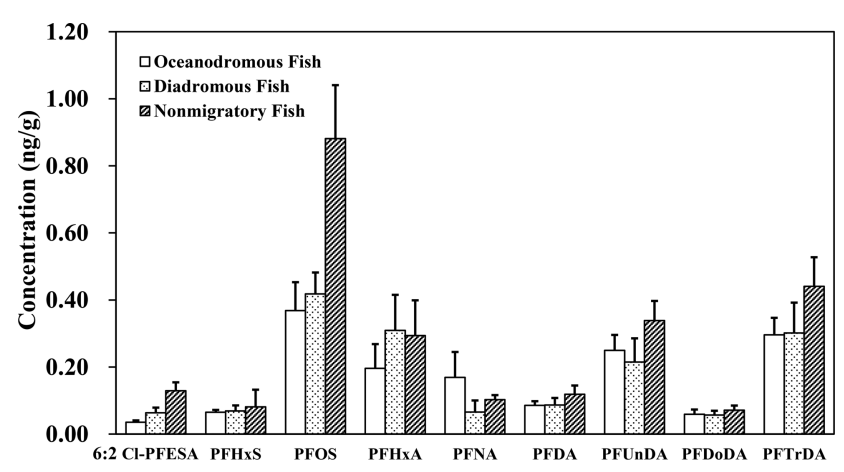

Figure 3. PFAS concentrations in different fishes with distinct migration behaviors.

of 6:2 Cl-PFESA in nonmigratory fish were significantly higher than those in oceanodromous fish (generalized Wilcoxon test, $p$ $<0.05)$. It was probably attributed to the different contamination status in the sea, as the concentrations of PFASs in ocean decreased with the increase of the distance from the coast. ${ }^{29,30}$ Similarly, Bohai Sea had heavier contamination, including Cl-PFESAs, than open sea. Therefore, the nonmigratory fish living in a heavier contaminated environment had higher levels of Cl-PFESAs than the oceanodromous fish. The concentrations of 6:2 Cl-PFESA in mantis shrimp were about 10 -fold higher than that in sand shrimp $(0.035 \pm 0.011 \mathrm{ng} / \mathrm{g}$; generalized Wilcoxon test, $p=$ 0.011). As for bivalves, venus clam contained a substantial amount of 6:2 Cl-PFESA $(0.037 \pm 0.007 \mathrm{ng} / \mathrm{g})$, in which the concentrations were comparable to those in some fishes and crustaceans. The great differences between the similar species might be attributed to their different diet habits and bioaccumulation potentials. Mantis shrimp stays at a higher trophic level than sand shrimp, and it preys on crustaceans, benthic mollusks and small fish. ${ }^{31}$ Extremely high levels of 6:2 Cl-PFESA in mantis shrimp and venus clam were consistent with previous findings that mantis shrimp and several mollusks had special bioaccumulation ability to PFASs and some other halogenated organic chemicals. ${ }^{17,31-35}$

Contribution of Cl-PFESAs to Total PFASs Burden in Marine Organisms. FTSAs were seldom detected in the marine biota samples because their usage as suppressants are at the trial phase, and they are mainly applied in aqueous filmforming foam (AFFF). ${ }^{1,36,37}$ It was reported that the degradation of FTSAs was easier than that of PFOS. ${ }^{38-40}$ The concentrations of PFSAs and PFCAs in marine organisms were comparable to previous findings in fish and invertebrates from Bohai Sea. ${ }^{34,41}$ The total concentrations of Cl-PFESAs $(<\mathrm{MDL}-0.612 \mathrm{ng} / \mathrm{g})$ were less than those of PFSAs $(<\mathrm{MDL}-$ $2.683 \mathrm{ng} / \mathrm{g})$ and PFCAs (<MDL-11.738 ng/g) in marine organisms $(p<0.01$, SI Figure S3), accounting for a small portion $(<10 \%)$ of the total PFASs burden.

6:2 Cl-PFESA was positively correlated with PFOS, PFNA, PFDA, PFUnDA, PFDoDA, and PFTrDA (Spearman's rho $=0.701,0.418,0.702,0.427,0.502$, and 0.532; $p<0.001)$. The total concentrations of Cl-PFESAs also displayed positive relationships with $\sum$ PFSAs and $\sum$ PFCAs (Spearman rho $=0.605$ and $0.339, p<0.001$ ). The correlations between $\mathrm{Cl}$ PFESAs and commonly concerned PFASs showed they had similar exposure sources and/or environmental behaviors. The relationships between lipid contents and chemical concentrations were also investigated, and the results showed there was no significant correlation between 6:2 Cl-PFESA concentrations and lipid contents (Spearman's rho $=0.200, p$ $=0.081)$, indicating that $6: 2$ Cl-PFESA did not tend to accumulate in the adipose. A recent tissue distribution study on crucian carps suggested that 6:2 Cl-PFESA preferentially bound with serum albumin and could be accumulated in blood. ${ }^{3}$ Other PFASs were also reported to be accumulated in protein-rich compartments (blood, liver, and kidney). ${ }^{14,42,43}$

Spatial Distribution and Temporal Variations of $\mathrm{Cl}-$ PFESAs. Studies on surface river water and sewage sludge samples indicated that the environmental Cl-PFESA concentrations in eastern coastal region were higher than those in some other places in China. ${ }^{1,7}$ For 9 rivers flowing into Bohai Sea, the highest level of 6:2 Cl-PFESA $(48.2 \mathrm{ng} / \mathrm{L})$ was found in the surface water samples from Hai River, and that in Yongding River $(12.2 \mathrm{ng} / \mathrm{L})$ came the second. ${ }^{7}$ The spatial variations among different sampling sites along the coastline were also investigated in the present study. According to the results of modified sign test, no significant spatial differences were found for Cl-PFESAs and the other compounds in marine organisms $(p>0.05)$, except that the concentrations of PFTrDA in samples from Dalian were significantly higher than those from Yantai $(p=0.007)$ and Penglai $(p=0.049)$, suggesting there was possible PFTrDA discharge at that sampling site.

The temporal variations of Cl-PFESAs were studied in mollusk samples (bivalve and gastropod) which were collected in 2010, 2012 and 2014. 6:2 Cl-PFESA was not detectable in mollusks collected in 2010 except for one venus clam sample. In our preliminary experiments, 6:2 Cl-PFESA was not found in the samples obtained in 2006, indicating a low detection frequency in mollusks sampled before 2010. The detection 
Table 1. TMF Values and Regression Parameters between Trophic Levels and Log Concentrations of 6:2 Cl-PFESA in Different Marine Organisms

\begin{tabular}{|c|c|c|c|c|c|c|c|}
\hline samples & intercept & $\overline{S E_{\text {Intercept }}}$ & slope & $\overline{S E_{\text {slope }}}$ & $R^{2}$ & $p$ value & TMF \\
\hline 2012, Dalian $(n=20)$ & 0.745 & 0.174 & 0.228 & 0.078 & 0.327 & 0.019 & 1.69 \\
\hline 2012 , all locations $(n=44)$ & 0.524 & 0.219 & 0.268 & 0.104 & 0.141 & 0.029 & 1.85 \\
\hline 2013, all locations $(n=35)$ & 0.888 & 0.394 & 0.363 & 0.140 & 0.170 & 0.014 & 2.31 \\
\hline 2014 , all locations $(n=27)$ & 1.505 & 1.065 & -0.234 & 0.494 & 0.014 & 0.635 & 0.58 \\
\hline all samples $(n=106)$ & 0.037 & 0.212 & 0.573 & 0.089 & 0.284 & $<0.001$ & 3.74 \\
\hline \multicolumn{8}{|c|}{ method 2: maximum likelihood estimation (MLE) } \\
\hline sample group & intercept & $S \mathrm{SE}_{\text {Intercept }}$ & slope & SE $_{\text {Slope }}$ & likelihood $r$ & $p$ value & TMF \\
\hline 2012, Dalian $(n=20)$ & 0.100 & 0.394 & 0.451 & 0.147 & 0.674 & $<0.001$ & 2.82 \\
\hline 2012, all locations $(n=44)$ & 0.164 & 0.298 & 0.407 & 0.130 & 0.446 & 0.002 & 2.55 \\
\hline 2013, all locations $(n=35)$ & 0.873 & 0.390 & 0.368 & 0.139 & 0.409 & 0.011 & 2.33 \\
\hline 2014, all locations $(n=27)$ & 1.640 & 0.826 & -0.213 & 0.386 & 0.106 & 0.580 & 0.61 \\
\hline all samples $(n=106)$ & -0.121 & 0.227 & 0.635 & 0.093 & 0.567 & $<0.001$ & 4.32 \\
\hline \multicolumn{8}{|c|}{ method 3: substitution the nondetectable data with half of MDL } \\
\hline sample group & intercept & $\mathrm{SE}_{\text {intercept }}$ & Slope & $\mathrm{SE}_{\text {slope }}$ & $R^{2}$ & $p$ value & TMF \\
\hline 2012, Dalian $(n=20)$ & 0.455 & 0.172 & 0.316 & 0.077 & 0.484 & $<0.001$ & 2.07 \\
\hline 2012, all locations $(n=44)$ & 0.550 & 0.165 & 0.261 & 0.079 & 0.207 & 0.002 & 1.82 \\
\hline 2013, all locations $(n=35)$ & 0.855 & 0.421 & 0.371 & 0.150 & 0.157 & 0.018 & 2.35 \\
\hline 2014, all locations $(n=27)$ & 1.578 & 0.677 & -0.151 & 0.315 & 0.009 & 0.636 & 0.71 \\
\hline all samples $(n=106)$ & 0.158 & 0.179 & 0.535 & 0.075 & 0.327 & $<0.001$ & 3.43 \\
\hline
\end{tabular}

frequencies of 6:2 Cl-PFESA in mollusks increased from $24 \%$ in $2012(\mathrm{n}=30)$ to $44 \%$ in $2014(\mathrm{n}=27)$. Among mollusk species with relatively high detection frequencies (venus clam, oyster and ark shell), 6:2 Cl-PFESA concentrations in samples collected in 2014 were significantly higher than those collected in $2012(p=0.022$, SI Figure S4). Meanwhile, 8:2 Cl-PFESA was only detectable in venus clam and ark shell collected in 2014. These temporal variations indicated Cl-PFESAs concentrations tended to increase in this region. Some kinds of mollusks are commonly used as bioindicators to reflect the contamination status of aquatic systems due to their wide existence and particularly high bioaccumulative potential. ${ }^{17,34,35}$ Considering its special bioaccumulative capability for the pollutants and the wide distribution, venus clam was hopefully to serve as a potential bioindicator for Cl-PFESA contamination in marine ecosystem.

Trophic Transfer in the Marine Food Web. Results from empirical research have substantiated that PFASs could be bioaccumulated and biomagnified along the freshwater and the marine food webs, posing a risk to ecosystem and human beings. ${ }^{10-15,21}$ Trophic transfer of Cl-PFESAs was studied here due to their similarities to PFASs in molecular structures, chemical properties, and the correlation with PFAS concentrations in marine organisms.

The trophic magnification factor (TMF) is usually applied to assess chemical transfer along with trophic levels. Biomagnification is generally indicated when TMFs are greater than 1 . Here, three regression analysis methods were applied in TMF calculation to avoid the biased results. ${ }^{27}$ As shown in Table 1, the TMF values of 6:2 Cl-PFESA in 20 marine biota samples collected from Dalian in $2012\left(p \leqslant 0.019, R^{2} \geqslant 0.327\right.$, TMF: 1.69-2.82) were greater than 1 , suggesting the biomagnification of 6:2 Cl-PFESA. However, as the sample scale was relatively small, the food web collection should be conducted across the locations of the top predator's foraging ranges. ${ }^{44}$ Considering the high movement of marine fish, trophic transfer of 6:2 Cl-PFESA was further studied using samples across
Bohai Sea including all sampling sites investigated in this study. The scatter plot of log concentrations of 6:2 Cl-PFESA, trophic levels and sampling time is presented in Figure 4. Significant linear correlations were found for the samples collected in both $2012\left(p \leq 0.029, R^{2} \geq 0.141\right)$, and $2013\left(p \leq 0.018, R^{2} \geq\right.$ 0.157 ) with the TMF values of $1.82-2.55$ and $2.31-2.35$, respectively (Table 1 ). The biomagnification of 6:2 Cl-PFESA

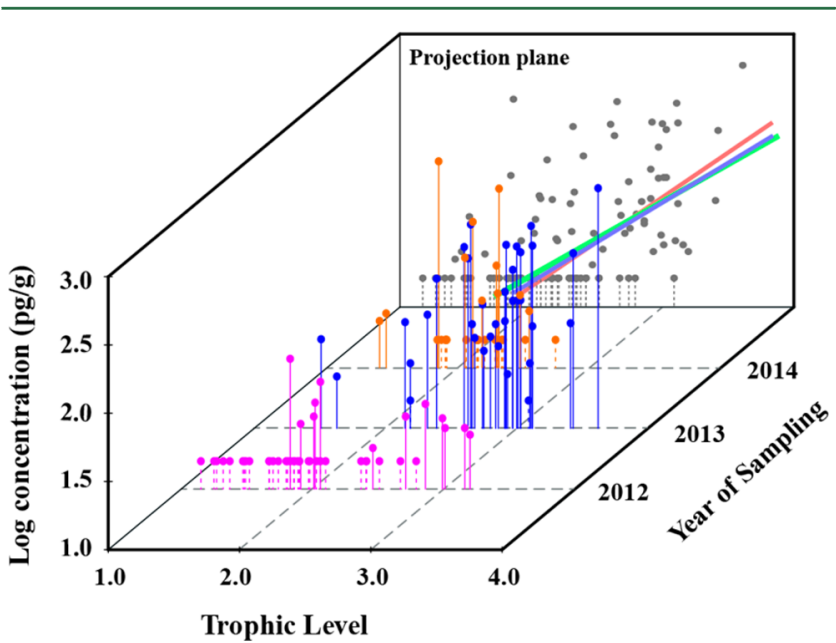

Figure 4. Scatter plot of log concentrations of 6:2 Cl-PFESA (pg/g) in marine organisms from Chinese Bohai Sea against trophic levels, and sampling time. Points with solid line represent detectable data. Points with dash line represent the MDLs of nondetectable data. 6:2 ClPFESA was detectable in 54\% of these samples from 2012 to 2014. All these data points were clustered on the projection plane. The green, blue, and red regression lines on the projection plane represent the regression results from simple substitution method $(1 / 2 \mathrm{MDL})$, the ROS method and MLE method. Substitution with 1/2MDL: TMF = 3.43, $p<0.001, R^{2}=0$ 0.327; Substitution with ROS-generated values: TMF $=3.74, p<0.001, R^{2}=0.284$; MLE method: TMF $=4.32, p<$ $0.001, R^{2}=0.567$. 
was thus verified on a large geographic scale. To evaluate $6: 2$ Cl-PFESA biomagnification behavior in all tested samples, the related data are clustered on the projection plane in Figure 4, and analyzed by three regression methods. Positive relationships were observed between 6:2 Cl-PFESA concentrations and TLs $\left(p \leq 0.001, R^{2} \geq 0.284\right.$, TMF: 3.43-4.32), further confirming the biomagnification of the chemical. The TMF values obtained by three methods were very close (Table1), and no significant interaction effect was found between TLs and the sampling years $(p<0.05)$. As for 8:2 Cl-PFESA, although its TMFs could not be calculated due to its low detection frequency, this compound probably had the similar bioaccumulation and biomagnification potentials because it had similar chemical structure to 6:2 Cl-PFESA.

Biomagnification of PFOS and long chain PFCAs ( $\mathrm{C}=9-$ 13) was also verified by the TMF values obtained by three regression approaches. As summarized in SI Table S6, it is accordant with the results from the literatures. ${ }^{11-15,45,46}$ TMFs of the commonly monitored PFASs in the present study were almost the same with those in the plankton-invertebrate-fish food web from Taihu Lake, China, while the values herein were lower than those estimated in Bohai Sea previously. ${ }^{12,13}$ The use of fish muscle, rather than whole body homogenates, was one of the possible reasons for this discrepancy. Additionally, migration behaviors of fish might affect the accurate evaluation of TMFs as well. ${ }^{44}$ 6:2 Cl-PFESA and some other PFASs were usually at low levels in oceanodromous fishes which were generally at high-trophic-level, but abundant in some lowtrophic-level mollusks which had special accumulation capacities, leading to negative skewness of TMF estimations to some extent. In comparison, the TMF values of $6: 2 \mathrm{Cl}$ PFESA (TMF: 3.43-4.32) were comparable with PFOS (TMF: 3.83-3.88) and PFTrDA (TMF: 3.54-4.40), higher than the other long chain PFCAs.

Concerns for Emerging Contamination of Cl-PFESAs. According to the limited environmental investigations, ClPFESAs seem to be omnipresent in various environmental matrices. ${ }^{1-3,6-8}$ Here we provided the first insight into their occurrence and trophic transfer in marine organisms from Bohai Sea, China. Although the concentrations of Cl-PFESAs in these organisms were generally lower than those of the other routinely monitored PFASs, the concentrations and detection frequencies of Cl-PFESAs in mollusks tended to increase within the sampling period from 2010 to 2014. Similar to PFASs, species-dependent concentration differences were observed for Cl-PFESAs, which were related with the trophic levels, dietary habits and migratory strategies of organisms. 6:2 Cl-PFESA concentrations in fish and invertebrates from Bohai Sea were comparable to the top predators of Arctic, which resulted from possible long-range transport, ${ }^{8}$ suggesting Cl-PFESA contamination was relatively higher in Bohai Sea than the Arctic. The prevalence and biomagnification potential of Cl-PFESAs in the marine ecosystem might help to increase public awareness on the ecological and health risks from the unintended exposure to this class of chemicals. Further studies on their toxicology, environmental fate, and potential health hazards are imperative. With the ongoing regulations on well-known hazardous PFASs, the related production and usage of their alternatives are increasing. Chemicals, like Cl-PFESAs, are facing lots of challenges, and need to be systematically studied before they are submitted to commercial process, regarding the concerns on their environmental behavior, ecological risks and human health issues.

\section{ASSOCIATED CONTENT}

\section{Supporting Information}

The Supporting Information is available free of charge on the ACS Publications website at DOI: 10.1021/acs.est.6b06593.

Species, sampling sites and time, sample size, lipid content (lipid\%), stable carbon $\left(\delta^{13} \mathrm{C}\right)$, stable nitrogen $\left(\delta^{15} \mathrm{~N}\right)$ and trophic levels (TLs) of marine samples from Bohai Sea, China (Table S1). The ions and isotopelabeled standards analyzed by LC-MS/MS for target chlorinated polyfluoroalkyl ether sulfonic acids (ClPFESAs), perfluorinated carboxylates (PFCAs), perfluoroalkyl sulfonates (PFSAs) and fluorotelomer sulfonates (FTSAs) (Table S2). Detection frequencies, method detection limits (MDLs) and concentrations (ng/g) of Cl-PFESAs, PFSAs and FTSAs in marine biota samples from Bohai Sea, China (Table S3). Detection frequencies, method detection limits (MDLs) and concentrations ( $\mathrm{ng} / \mathrm{g}$ ) of perfluorinated carboxylates (PFCAs) in marine samples from Bohai Sea, China (Table S4). TMF values and regression parameters between trophic levels and log concentrations of PFOS and long chain PFCAs in marine organisms from Bohai Sea, China (Table S5). Trophic magnification factors (TMFs) of PFASs from the references (Table S6). The sampling locations around Bohai Sea, China (Figure S1). The molecular structures of target analytes (Figure S2). The total concentrations of Cl-PFESAs, PFSAs and PFCAs in different marine organisms from Bohai Sea, China (Figure S3). The levels of 6:2 Cl-PFESA in oyster, ark shell and venus clam collected in 2012 and 2014 (Figure S4) (PDF)

\section{AUTHOR INFORMATION}

\section{Corresponding Author}

*Phone 8610-62849334; fax: 8610-62849339; e-mail: liujy@ rcees.ac.cn.

ORCID $\odot$

Ting Ruan: 0000-0002-6222-374X

Yawei Wang: 0000-0002-6115-4076

Jiyan Liu: 0000-0002-7553-3325

\section{Notes}

The authors declare no competing financial interest.

\section{ACKNOWLEDGMENTS}

This work was jointly supported by the National Basic Research Program of China (2014CB441105), National Natural Science Foundation of China (21177147, 21577151, 21621064) and the Strategic Priority Research Program of the Chinese Academy of Sciences (XDB14010400).

\section{REFERENCES}

(1) Ruan, T.; Lin, Y.; Wang, T.; Liu, R.; Jiang, G. Identification of novel polyfluorinated ether sulfonates as PFOS alternatives in municipal sewage sludge in China. Environ. Sci. Technol. 2015, 49 (11), 6519-6527.

(2) Wang, S.; Huang, J.; Yang, Y.; Hui, Y.; Ge, Y.; Larssen, T.; Yu, G.; Deng, S.; Wang, B.; Harman, C. First report of a Chinese PFOS alternative overlooked for 30 years: Its toxicity, persistence, and presence in the environment. Environ. Sci. Technol. 2013, 47 (18), 10163-10170.

(3) Shi, Y.; Vestergren, R.; Zhou, Z.; Song, X.; Xu, L.; Liang, Y.; Cai, $\mathrm{Y}$. Tissue distribution and whole body burden of the chlorinated 
polyfluoroalkyl ether sulfonic acid F-53B in crucian carp (Carassius carassius): Evidence for a highly bioaccumulative contaminant of emerging concern. Environ. Sci. Technol. 2015, 49 (24), 14156-14165.

(4) Gomis, M. I.; Wang, Z.; Scheringer, M.; Cousins, I. T. A modeling assessment of the physicochemical properties and environmental fate of emerging and novel per- and polyfluoroalkyl substances. Sci. Total Environ. 2015, 505, 981-991.

(5) Shanghai Guangming Electroplating Plant; Shanghai Institute of Organic Chemistry at Chinese Academy of Sciences. Jiangsu Taizhou Electrochemical Plant. Preparation of F-53 and its application in chrome mist suppression. Mater. Prot. (in Chinese) 1976, 3 (3), 27-32.

(6) Shi, Y.; Vestergren, R.; Xu, L.; Zhou, Z.; Li, C.; Liang, Y.; Cai, Y. Human exposure and elimination kinetics of chlorinated polyfluoroalkyl ether sulfonic acids (Cl-PFESAs). Environ. Sci. Technol. 2016, 50 (5), 2396-2404.

(7) Wang, T.; Vestergren, R.; Herzke, D.; Yu, J.; Cousins, I. T. Levels, isomer profiles and estimated riverine mass discharges of perfluoroalkyl acids and fluorinated alternatives at the mouths of Chinese rivers. Environ. Sci. Technol. 2016, 50 (21), 11584-11592.

(8) Gebbink, W. A.; Bossi, R; Rigét, F. F.; Rosing-Asvid, A.; Sonne, C.; Dietz, R. Observation of emerging per- and polyfluoroalkyl substances (PFASs) in Greenland marine mammals. Chemosphere 2016, 144, 2384-2391.

(9) Zhang, Q.; Liu, W.; Niu, Q.; Wang, Y.; Zhao, H.; Zhang, H. Effects of perfluorooctane sulfonate and its alternatives on long-term potentiation in the hippocampus CA1 region of adult rats in vivo. Toxicol. Res. (Cambridge, U. K.) 2016, 5, 539-546.

(10) Conder, J. M.; Hoke, R. A.; Wolf, W. D.; Russell, M. H.; Buck, R. C. Are PFCAs bioaccumulative? A critical review and comparison with regulatory criteria and persistent lipophilic compounds. Environ. Sci. Technol. 2008, 42 (4), 995-1003.

(11) Loi, E. I. H.; Yeung, L. W. Y.; Taniyasu, S.; Lam, P. K. S.; Kannan, K.; Yamashita, N. Trophic magnification of poly- and perfluorinated compounds in a subtropical food web. Environ. Sci. Technol. 2011, 45 (13), 5506-5513.

(12) Fang, S.; Chen, X.; Zhao, S.; Zhang, Y.; Jiang, W.; Yang, L.; Zhu, L. Trophic magnification and isomer fractionation of perfluoroalkyl substances in the food web of Taihu Lake, China. Environ. Sci. Technol. 2014, 48 (4), 2173-82.

(13) Zhang, Z.; Peng, H.; Wan, Y.; Hu, J. Isomer-specific trophic transfer of perfluorocarboxylic acids in the marine food web of Liaodong Bay, North China. Environ. Sci. Technol. 2015, 49 (3), 14531461.

(14) Kelly, B. C.; Ikonomou, M. G.; Blair, J. D.; Surridge, B.; Hoover, D.; Grace, R.; Gobas, F. A. P. C. Perfluoroalkyl contaminants in an Arctic marine food web. Environ. Sci. Technol. 2009, 43 (15), 40374043.

(15) Martin, J. W.; Whittle, D. M.; Muir, D. C. G.; Mabury, S. A. Perfluoroalkyl contaminants in a food web from Lake Ontario. Environ. Sci. Technol. 2004, 38 (20), 5379-5385.

(16) Meng, M.; Shi, J.; Liu, C.; Zhu, N.; Shao, J.; He, B.; Cai, Y.; Jiang, G. Biomagnification of mercury in mollusks from coastal areas of the Chinese Bohai Sea. RSC Adv. 2015, 5 (50), 40036-40045.

(17) Zhu, N.; Li, A.; Wang, T.; Wang, P.; Qu, G.; Ruan, T.; Fu, J.; Yuan, B.; Zeng, L.; Wang, Y.; Jiang, G. Tris(2,3-dibromopropyl) isocyanurate, hexabromocyclododecanes, and polybrominated diphen$\mathrm{yl}$ ethers in mollusks from Chinese Bohai Sea. Environ. Sci. Technol. 2012, 46 (13), 7174-7181.

(18) Zhou, Z.; Liang, Y.; Shi, Y.; Xu, L.; Cai, Y. Occurrence and transport of perfluoroalkyl acids (PFAAs), including short-chain PFAAs in Tangxun Lake, China. Environ. Sci. Technol. 2013, 47 (16), 9249-9257.

(19) Logan, J. M.; Jardine, T. D.; Miller, T. J.; Bunn, S. E.; Cunjak, R. A.; Lutcavage, M. E. Lipid corrections in carbon and nitrogen stable isotope analyses: Comparison of chemical extraction and modelling methods. J. Anim. Ecol. 2008, 77 (4), 838-846.

(20) Yi, W.; Jianying, H.; Lihui, A.; Wei, A.; Min, Y.; Mitsuaki, I.; Tatsuya, H.; Shu, T. Determination of trophic relationships within a
Bohai Bay food web using stable $\delta^{15} \mathrm{~N}$ and $\delta^{13} \mathrm{C}$ analysis. Chin. Sci. Bull. 2005, 50 (10), 1021-1025.

(21) Tomy, G. T.; Budakowski, W.; Halldorson, T.; Helm, P. A.; Stern, G. A.; Friesen, K.; Pepper, K.; Tittlemier, S. A.; Fisk, A. T. Fluorinated organic compounds in an eastern Arctic marine food web. Environ. Sci. Technol. 2004, 38 (24), 6475-6481.

(22) Haug, L. S.; Thomsen, C.; Brantsæter, A. L.; Kvalem, H. E.; Haugen, M.; Becher, G.; Alexander, J.; Meltzer, H. M.; Knutsen, H. K. Diet and particularly seafood are major sources of perfluorinated compounds in humans. Environ. Int. 2010, 36 (7), 772-778.

(23) Post, D. M. Using stable isotopes to estimate trophic position: Models, methods, and assumptions. Ecology 2015, 83 (3), 703-718.

(24) Fisk, A. T.; Hobson, K. A.; Norstrom, R. J. Influence of chemical and biological factors on trophic transfer of persistent organic pollutants in the Northwater Polynya marine food web. Environ. Sci. Technol. 2001, 35 (4), 732-738.

(25) Hop, H.; Borgå, K.; Gabrielsen, G. W.; Kleivane, L.; Skaare, J. U. Food web magnification of persistent organic pollutants in poikilotherms and homeotherms from the Barents Sea. Environ. Sci. Technol. 2002, 36 (12), 2589-2597.

(26) Helsel, D. R. Statistics for Censored Environmental Data Using Minitab and R; John Wiley \& Sons, 2011; Vol. 77.

(27) Borgå, K.; Kidd, K. A.; Muir, D. C. G.; Berglund, O.; Conder, J. M.; Gobas, F. A. P. C.; Kucklick, J.; Malm, O.; Powellkk, D. E. Trophic magnification factors: Considerations of ecology, ecosystems, and study design. Integr. Environ. Assess. Manage. 2012, 8 (1), 64-84.

(28) FishBase Website. http://www.fishbase.org.

(29) Takemine, S.; Matsumura, C.; Yamamoto, K.; Suzuki, M.; Tsurukawa, M.; Imaishi, H.; Nakano, T.; Kondo, A. Discharge of perfluorinated compounds from rivers and their influence on the coastal seas of Hyogo prefecture, Japan. Environ. Pollut. 2014, 184, 397-404.

(30) Ahrens, L.; Felizeter, S.; Ebinghaus, R. Spatial distribution of polyfluoroalkyl compounds in seawater of the German Bight. Chemosphere 2009, 76 (2), 179-184.

(31) Tian, S.; Zhu, L.; Liu, M. Bioaccumulation and distribution of polybrominated diphenyl ethers in marine species from Bohai Bay, China. Environ. Toxicol. Chem. 2010, 29 (10), 2278-2285.

(32) Gulkowska, A.; Jiang, Q.; So, M. K.; Taniyasu, S.; Lam, P. K. S.; Yamashita, N. Persistent perfluorinated acids in seafood collected from two cities of China. Environ. Sci. Technol. 2006, 40 (12), 3736-3741.

(33) Chen, C.; Wang, T.; Naile, J. E.; Li, J.; Geng, J.; Bi, C.; Hu, W.; Zhang, X.; Khim, J. S.; Feng, Y.; Giesy, J. P.; Lu, Y. Perfluorinated compounds in aquatic products from Bohai Bay, Tianjin, China. Hum. Ecol. Risk Assess. 2011, 17 (6), 1279-1291.

(34) Pan, Y.; Shi, Y.; Wang, Y.; Cai, Y.; Jiang, G. Investigation of perfluorinated compounds (PFCs) in mollusks from coastal waters in the Bohai Sea of China. J. Environ. Monit. 2010, 12 (2), 508-513.

(35) Wang, Y.; Wang, T.; Li, A.; Fu, J.; Wang, P.; Zhang, Q.; Jiang, G. Selection of bioindicators of polybrominated diphenyl ethers, polychlorinated biphenyls, and organochlorine pesticides in mollusks in the Chinese Bohai Sea. Environ. Sci. Technol. 2008, 42 (19), 71597165.

(36) UNEP. Technical Paper on the Identification and Assessment of Alternatives to the Use of Perfluorooctane Sulfonic Acid in Open Applications (UNEP/POPS/POPRC.8/INF/17); Stockholm Convention on Persistent Organic Pollutants, United Nations Environment Programme, 2012.

(37) Backe, W. J.; Day, T. C.; Field, J. A. Zwitterionic, cationic, and anionic fluorinated chemicals in aqueous film forming foam formulations and groundwater from U.S. military bases by nonaqueous large-volume injection HPLC-MS/MS. Environ. Sci. Technol. 2013, 47 (10), 5226-5234.

(38) Yang, X.; Huang, J.; Zhang, K.; Yu, G.; Deng, S.; Wang, B. Stability of 6:2 fluorotelomer sulfonate in advanced oxidation processes: Degradation kinetics and pathway. Environ. Sci. Pollut. Res. 2014, 21 (6), 4634-4642.

(39) Wang, N.; Liu, J.; Buck, R. C.; Korzeniowski, S. H.; Wolstenholme, B. W.; Folsom, P. W.; Sulecki, L. M. 6:2 Fluorotelomer 
sulfonate aerobic biotransformation in activated sludge of waste water treatment plants. Chemosphere 2011, 82 (6), 853-858.

(40) Jin, L.; Jiang, C.; Zhang, P. Photochemical decomposition of $1 \mathrm{H}, 1 \mathrm{H}, 2 \mathrm{H}, 2 \mathrm{H}$-perfluorooctane sulfonate (6:2FTS) induced by ferric ions. J. Environ. Sci. 2016, 51, 120-127.

(41) Yang, L.; Tian, S.; Zhu, L.; Liu, Z.; Zhang, Y. Bioaccumulation and distribution of perfloroalkyl acids in seafood products from Bohai Bay, China. Environ. Toxicol. Chem. 2012, 31 (9), 1972-1979.

(42) Guy, W. S.; Taves, D. R.; Brey, W. S., Jr. Organic fluorocompounds in human plasma: Prevalence and characterization. Biochemistry Involving Carbon-Fluorine Bonds; ACS Symposium 1976, 28, 117.

(43) Martin, J. W.; Mabury, S. A.; Solomon, K. R.; Muir, D. C. G. Dietary accumulation of perfluorinated acids in juvenile rainbow trout (Oncorhynchus mykiss). Environ. Toxicol. Chem. 2003, 22 (1), 196-204.

(44) McLeod, A. M.; Arnot, J. A.; Borgå, K.; Selck, H.; Kashian, D. R.; Krause, A.; Paterson, G.; Haffner, G. D.; Drouillard, K. G. Quantifying uncertainty in the trophic magnification factor related to spatial movements of organisms in a food web. Integr. Environ. Assess. Manage. 2015, 11 (2), 306-318.

(45) Houde, M.; Bujas, T. A. D.; Small, J.; Wells, R. S.; Fair, P. A.; Bossart, G. D.; Solomon, K. R.; Muir, D. C. G. Biomagnification of perfluoroalkyl compounds in the bottlenose dolphin (Tursiops truncatus) food web. Environ. Sci. Technol. 2006, 40 (13), 4138-4144.

(46) Houde, M.; Czub, G.; Small, J. M.; Backus, S.; Wang, X.; Alaee, M.; Muir, D. C. G. Fractionation and bioaccumulation of perfluorooctane sulfonate (PFOS) isomers in a Lake Ontario food web. Environ. Sci. Technol. 2008, 42 (24), 9397-9403. 